\title{
The Effect of Noise Correlation on Fractional Sampling based Spectrum Sensing
}

\author{
Shree Krishna Sharma*, Symeon Chatzinotas*, Björn Ottersten ${ }^{* \dagger}$ \\ * SnT - securityandtrust.lu, University of Luxembourg \\ \{shree.sharma, symeon.chatzinotas, bjorn.ottersten\}@uni.lu \\ ${ }^{\dagger}$ Royal Institute of Technology (KTH), Sweden, Email: bjorn.ottersten@ee.kth.se
}

\begin{abstract}
This paper considers a Fractional Sampling (FS) technique to enhance the Spectrum Sensing (SS) efficiency of a Cognitive Radio (CR) using a decision statistic based on asymptotic Random Matrix Theory (RMT). Firstly, the effect of noise correlation on eigenvalue based SS is studied analytically and by numerical evaluation. Secondly, new bounds for the Standard Condition Number (SCN) are proposed to enhance the SS efficiency in correlated noise scenarios. It is shown that proposed FS method can enhance SS efficiency up to certain FS rates at the expense of receiver complexity and no performance advantage is obtained if the FS rate is increased beyond this limit. As a result, a method for determining the operating point for the FS rate in terms of sensing performance and complexity is suggested.
\end{abstract}

Index Terms-Spectrum Sensing, Fractional Sampling, Eigenvalue Distribution, Noise Correlation

\section{INTRODUCTION}

Due to rapidly increasing need for higher data rates, the demand for radio frequency spectrum is increasing while the available radio frequency spectrum is becoming scarce due to current static frequency allocation schemes. However, different spectrum occupancy measurement campaigns carried out at different parts of the world show that the average spectrum occupancy rate is very low [1] and it shows spatial as well as temporal variations. In this aspect, innovative techniques to exploit the opportunistic usage of underutilized spectrum are needed. Cognitive Radio (CR), which can exploit the unused licensed spectrum opportunistically in secondary way, has been considered as a potential candidate to increase the spectral efficiency in future wireless systems [2,3]. Spectrum Sensing (SS) is an important mechanism in order to acquire the spectrum awareness required by the CRs. Several SS techniques have been proposed in the literature for sensing the presence of a Primary User (PU) [4-6]. Matched filter and cyclostationary feature detection techniques require the prior knowledge of the PU's signal to make the decision about the presence or absence of the PU signal [2]. Although Energy Detection (ED) technique does not require any prior knowledge of PU's signal, the performance of this technique is susceptible to noise covariance uncertainty [7]. Due to the absence of prior knowledge about channel, PU signal and noise variance in practical scenarios, exploring efficient blind SS techniques has been an important research challenge.

Furthermore, the performance of traditional SS techniques may be severely degraded in multi-path fading and shadowing environments. Different diversity enhancing techniques such as multi-antenna, cooperative and oversampled techniques have been introduced in the literature to enhance the SS efficiency in wireless fading channels [8-10]. In most of these methods, the properties of the eigenvalues of the received signal's covariance matrix have been considered using the recent results from advances in Random Matrix Theory (RMT) $[11,12]$. Eigenvalue based SS technique does not require any prior information of the PU's signal and it outperforms ED techniques, especially in the presence of noise covariance uncertainty [8].

Several eigenvalue based SS methods utilizing the properties of the eigenvalues of Wishart random matrices have been proposed in $[8,10,13]$. Wishart random matrices appear in the analysis under noise only cases and in white noise scenarios. The authors in [13] use Marchenko-Pastur (MP) law to test a binary hypothesis assuming the presence of white noise. However, the noise covariance matrix does not follow the properties of Wishart random matrix in the presence of correlated noise [8]. In practical situations, different phenomena such as oversampling and imperfections in filtering may cause noise correlation [8]. For example, when a received signal is filtered by a narrowband receive filter, the noise embedded in the received signal is also filtered and the output signal of the filter contains the correlated noise. In the presence of noise correlation, the eigenvalue distribution does not follow the MP law and the threshold proposed in [13] may not provide better sensing performance. Therefore, new sensing thresholds need to be investigated to carry out SS in the presence of noise correlation. In this direction, firstly, we use the Standard Condition Number (SCN) of the noise covariance matrix to analyze the effect of noise correlation in eigenvalue based SS techniques and then the SCN of the received signal's covariance matrix for decision process. The SCN of a matrix is defined as the ratio of the maximum eigenvalue to the minimum eigenvalue [14] and can be used as a metric to characterize the support of the asymptotic eigenvalue probability distribution function (a.e.p.d.f.) of a random matrix. If the calculated SCN is greater than noise only SCN, it is decided that a PU signal is present. Since noise correlation affects the $\mathrm{SCN}$ of the noise covariance matrix and as a result, the SCN of the received signal's covariance matrix, the decision metric is also affected. According to author's knowledge, the analysis of eigenvalue based SS in the presence of noise correlation 
using this concept has not been considered in the literature. This is the first contribution of this paper.

Moreover, we analyze the performance of Fractional Sampling (FS) based SS technique in this paper. The sampling rate in the receiver can be increased beyond the symbol rate, known as FS, to enhance the SS efficiency under fading channel conditions. FS technique is commonly used to enhance signal detection reliability in the receiver $[15,16]$. From the $C R$ point of view, a FS based receiver can be modeled as a virtual multiple-output system with presumably independent channel fading effects. This technique is especially beneficial in time varying channels with small channel coherence time. Another motivation factor for introducing the FS concept in the context of CR is that using more antennas at the receive-side is often impractical and expensive requiring multiple RF chains. FS introduces diversity and can improve the sensing efficiency of the PU's signal in wireless fading environments. However, it also results in colored noise when the bandwidth of receive filter is not sufficiently large [17] and this phenomenon gradually saturates the performance gain due to FS [15]. Therefore, it is important to determine the operating point for the FS rate, a design parameter that we can actually configure to find a good trade-off between performance and complexity. This is the second contribution of this paper.

The remainder of this paper is structured as follows: Section II provides considered signal model and noise correlation modeling used in this paper. Section III analyzes the effect of noise correlation on eigenvalue based SS for the noise only case. Section IV presents the proposed SCN-based decision statistic for FS scenario. Section V studies the performance of eigenvalue based SS technique in the presence of noise correlation with numerical simulations and proposes a method for determining the optimal FS operating point. Section VI concludes the paper.

\section{A. Notation}

Throughout the formulations of this paper, $\mathbb{E}[\cdot]$ denotes expectation, $(\cdot)^{T}$ denotes the transpose matrix, $(\cdot)^{H}$ denotes the conjugate transpose matrix, $(\cdot)^{*}$ represents the complex conjugate, $\operatorname{abs}(\cdot)$ denotes the absolute value, $\mathcal{S}_{\mathbf{X}}$ represents Stieltjes transform of $\mathbf{X}$, and $\Sigma_{\mathbf{X}}$ represents $\Sigma$ transform [11].

\section{Signal MOdel}

Let $N$ be the number of samples analyzed by the cognitive user for the decision process in the time duration of $\tau$ while performing symbol rate sampling. We consider a single cognitive user and a single primary user in our analysis. The sampling rate in the receiver can be increased beyond the symbol rate to enhance the signal detection capability in wireless fading channels. This technique, known as FS [15], produces $N$ FS samples out of each original sample. Let $M$ be the FS rate applied at the input of cognitive receiver. From signal model point of view, this factor can be considered as the number of multiple outputs analogous to the number of cooperating users in cooperative based sensing or the number of antennas in multiple antenna sensing as considered in related literature $[8,13]$. We denote the hypotheses of the presence and absence of the PU signal by $H_{1}$ and $H_{0}$ respectively. A binary hypothesis testing problem for $k$-th FS branch, $k=1, \ldots, M$, can be written as:

$$
\begin{aligned}
& H_{0}: y_{k}(i)=\hat{z}_{k}(i) \quad \text { PU absent } \\
& H_{1}: y_{k}(i)=h_{k}(i) s(i)+\hat{z}_{k}(i) \quad \text { PU present }
\end{aligned}
$$

where $y_{k}(i)$ is the signal observed by a $k$-th receiving dimension at $i$-th instant, $i=1,2, . ., N, s(i)$ is the PU signal at $i$-th instant, $h_{k}(i)$ is the amplitude gain of the channel for $k$-th receive dimension at $i$-th instant, and $\hat{z}_{k}(i)$ denotes the colored noise for $k$-th receive dimension at $i$-th instant. For our analysis, we assume that transmitted symbols are independent and identically distributed (i.i.d.) complex circularly symmetric (c.c.s.) Gaussian symbols, the noise samples in each FS branch are independent but are correlated across FS branches. The $M \times N$ channel matrix $\mathbf{H}$ consists of i.i.d. coefficients and each row of $\mathbf{H}$ represents the channel coefficients for $N$ number of samples for each FS branch i.e., $\mathbf{H} \triangleq\left[\mathbf{h}_{1}^{T}, \mathbf{h}_{2}^{T}, \ldots, \mathbf{h}_{M}^{T}\right]^{T}$, with $\mathbf{h}_{m} \triangleq\left[\begin{array}{llll}h_{m}(1) & h_{m}(2) & \ldots & h_{m}(N)\end{array}\right]$ with $m=$ $1,2, \ldots, M$. We assume channel coefficients to be i.i.d. in each FS branch and the channel coherence time to be sufficiently small so that channel is not correlated as we increase the FS rate. The sensing duration $(\tau)$ and symbol interval $\left(T_{s}\right)$ may not be the same depending upon the signal bandwidth and sampling rate used at the cognitive receiver. For example, let us consider a coexistence scenario of TV whitespace broadband and wireless microphone systems. These are two systems with different operation bandwidths, a microphone signal typically occupies $200 \mathrm{kHz}$ bandwidth while TV signal occupies $6 \mathrm{MHz}$ and microphone operates on TV bands [8]. In this scenario, $\tau$ becomes much greater than $T_{s}$. We consider the following signal models considering the relation between $\tau$ and $T_{s}$ under the $H_{1}$ hypothesis.

Case 1: In this case, the transmitted symbol remains constant during the sensing period. The received signal matrix in this case can be written as: $\mathbf{Y}=\sqrt{p} \mathbf{H} s+\hat{\mathbf{Z}}$, where $s$ is a constant transmitted symbol, $p$ is the power of transmitted symbol and $\hat{\mathbf{Z}} \triangleq\left[\hat{\mathbf{z}}_{1}^{T}, \hat{\mathbf{z}}_{2}^{T}, \ldots, \hat{\mathbf{z}}_{M}^{T}\right]^{T}$, with $\hat{\mathbf{z}}_{m} \triangleq$ $\left[\begin{array}{llll}\hat{z}_{m}(1) & \hat{z}_{m}(2) & \ldots & \hat{z}_{m}(N)\end{array}\right]$. The sample covariance of transmitted signal in this case can be written as: $R_{s}=\mathbb{E}\left[s^{2}\right]=$ 1.

Case 2: In this case, each fractional sampled branch i.e., each row of matrix $\mathbf{Y}$ includes the samples for a single symbol. $\mathbf{Y}=\sqrt{p} \mathbf{H} \mathbf{S}_{d}+\hat{\mathbf{Z}}$, where $\mathbf{S}_{d}$ is the diagonal transmitted signal matrix of dimension $N \times N$ with diagonal $\mathbf{s}=[s(1) \ldots s(N)]$. In this case, the sample covariance matrix of the transmitted signal becomes

$\mathbf{R}_{\mathbf{S}}=\mathbb{E}\left[\mathbf{S}_{d} \mathbf{S}_{d}^{H}\right]=\left[\begin{array}{cccc}\mathbb{E}\left[s^{2}(1)\right] & 0 & \cdots & 0 \\ 0 & \mathbb{E}\left[s^{2}(2)\right] & \cdots & 0 \\ & & \ddots & \\ 0 & 0 & \cdots & \mathbb{E}\left[s^{2}(N)\right]\end{array}\right]=\mathbf{I}$ (2)

assuming that for each sample we get an i.i.d. c.c.s. Gaussian symbol with $\mathbb{E}\left[s^{2}\right]=1$. 
In both the above cases, the $M \times N$ received signal matrix $\mathbf{Y}$ can be written in the following form.

$$
\mathbf{Y}=\left[\begin{array}{c}
\mathbf{y}_{1} \\
\mathbf{y}_{2} \\
\vdots \\
\mathbf{y}_{M}
\end{array}\right]=\left[\begin{array}{cccc}
y_{1}(1) & y_{1}(2) & \ldots & y_{1}(N) \\
y_{2}(1) & y_{2}(2) & \ldots & y_{2}(N) \\
\vdots & \vdots & \ddots & \vdots \\
y_{M}(1) & y_{M}(2) & \ldots & y_{M}(N)
\end{array}\right]
$$

$\mathbf{Y}$ can be further written as:

$$
\mathbf{Y}= \begin{cases}\sqrt{p} \mathbf{H} s+\hat{\mathbf{Z}}, & \text { Case } 1 \\ \sqrt{p} \mathbf{H} \mathbf{S}_{d}+\hat{\mathbf{Z}}, & \text { Case } 2\end{cases}
$$

where $\hat{\mathbf{Z}} \sim \mathcal{C N}\left(0, \hat{\mathbf{R}}_{\hat{\mathbf{Z}}}(N)\right)$ is the colored noise. The $\mathrm{SCN}$ of $\hat{\mathbf{R}}_{\hat{\mathbf{Z}}}(N)$ depends on the noise correlation among noise samples across FS branches. Assuming that the source signal is independent from the noise, the covariance matrix of received signal, $\mathbf{R}_{\mathbf{Y}}$, can be written as [8]:

$$
\begin{aligned}
\mathbf{R}_{\mathbf{Y}} & =\mathbb{E}\left[\mathbf{Y} \mathbf{Y}^{H}\right]=\mathbb{E}\left[(\sqrt{p} \mathbf{H S})(\sqrt{p} \mathbf{H S})^{H}\right]+\mathbb{E}\left[\hat{\mathbf{Z}} \hat{\mathbf{Z}}^{H}\right] \\
& =p \mathbf{H} \mathbf{H}^{H}+\mathbf{R}_{\hat{\mathbf{Z}}}
\end{aligned}
$$

where $\mathbf{R}_{\hat{\mathbf{Z}}}=\mathbb{E}\left[\hat{\mathbf{Z}} \hat{\mathbf{Z}}^{H}\right]$. Let us define sample covariance matrices of received signal and noise as: $\hat{\mathbf{R}}_{\mathbf{Y}}(N)=\frac{1}{N} \mathbf{Y} \mathbf{Y}^{H}$ and $\hat{\mathbf{R}}_{\hat{\mathbf{Z}}}(N)=\frac{1}{N} \hat{\mathbf{Z}} \hat{\mathbf{Z}}^{H}$. Since our detection method only depends on $\hat{\mathbf{R}}_{z}(N)$ as described in section III, all the signal types can be considered under this method.

\section{A. Noise Correlation Modeling}

A simple correlation model can be employed to analyze the noise correlation effect mathematically. In this work, we consider one-sided noise correlation model. Using this model, the colored noise can be related to the white noise using the following expression.

$$
\hat{\mathbf{Z}}=\boldsymbol{\Theta}^{1 / 2} \mathbf{Z}
$$

where $\Theta$ is an $M \times M$ Hermitian matrix whose entries correspond to the correlation among noise samples across FS branches and $\mathbf{Z}$ is an $M \times N$ matrix with c.c.s. i.i.d. Gaussian entries with zero mean and unit variance, representing the white noise. To ensure that $\Theta$ does not affect the noise power, we consider the following normalization:

$$
(1 / M) \operatorname{trace}\{\boldsymbol{\Theta}\}=1
$$

SS related papers mostly consider baseband processing and white noise scenarios without including the effect of the filter on the received signal. However, in practical implementation of a $\mathrm{CR}$, the received signal should be passed through a pulse shaping filter before further processing and thus noise is no longer white. The transfer function of the pulse shaping filter used at the input of RF front end of a CR affects the noise covariance matrix. Due to absence of channel knowledge and PU signal, coherent receivers such as matched filter are not suitable for the SS application. Active RC filters with tunable cut off frequencies has been proposed in the literature for CR applications $[18,19]$. When a white noise input process $n(t)$ with power spectral density $N_{0} / 2$ is the input to a $\mathrm{RC}$ filter with time constant $\mathrm{RC}$, the noise becomes colored after filtering. Although the channel may also get correlated at the output of the filter, we are interested in analyzing the effect of noise correlation on SS performance in this work assuming that noise correlation effect dominates the overall effect. Since the autocorrelation function of output process of RC filter resembles the exponential model, we consider exponential correlation to model $\Theta$ in this work. The exponential correlation model can be written as [20,21]:

$$
\theta_{i j}= \begin{cases}\rho^{(j-i)}, & i \leq j \\ \left(\rho^{(i-j)}\right)^{*}, & i>j\end{cases}
$$

where $\theta_{i j}$ is the $(i, j)$-th element of $\Theta$ and $\rho \in \mathcal{C}$ is the correlation coefficient with $|\rho| \leq 1$.

\section{NoISE CORRELATION ANALYSIS USING RMT}

RMT has been used in the literature in various applications such as modeling multiuser MIMO fading [21] and transmit/receive correlation in MIMO channels [22]. In this section, we present the theoretical analysis of the effect of noise correlation in the performance of eigenvalue based SS technique under $H_{0}$ hypothesis using RMT. Similar analysis under $H_{1}$ hypothesis has been investigated in [23] for Signal to Noise Ratio (SNR) estimation of the PU signal in the presence of noise correlation. Herein, we state two RMT based theorems which we are going to use in defining our decision statistics.

Theorem 3.1: [11] Consider an $M \times N$ matrix $\mathbf{F}$ whose entries are independent zero-mean complex (or real) random variables with variance $\frac{1}{N}$ and fourth moments of order $O\left(\frac{1}{N^{2}}\right)$. As $M, N \rightarrow \infty$ with $\frac{N}{M} \rightarrow \beta$, the empirical distribution of the eigenvalues of $\frac{1}{N} \mathbf{F F}^{H}$ converges almost surely to a non random limiting distribution with density given by:

$$
f_{\beta}(\lambda)=\left(1-\frac{1}{\beta}\right)^{+} \delta(\lambda)+\frac{\sqrt{(\lambda-a)^{+}(b-\lambda)^{+}}}{2 \pi \beta \lambda}
$$

where $(z)^{+}$denotes the $\max (0, z), a=(1-\sqrt{\beta})^{2}, b=$ $(1+\sqrt{\beta})^{2}$. The parameters $a$ and $b$ define the support of the distribution and correspond to the minimum eigenvalue $\left(\lambda_{\min }\right)$ and the maximum eigenvalue $\left(\lambda_{\max }\right)$ respectively and the ratio $b / a$ defines the SCN of $\frac{1}{N} \mathbf{F F}{ }^{H}$. The above limiting distribution is the MP law with ratio index $\beta$.

In practice, only a finite number of samples are available and the sample covariance matrix $\hat{\mathbf{R}}_{\mathbf{Y}}(N)$ may deviate from the statistical covariance matrix $\mathbf{R}_{\mathbf{Y}}$ [8]. Due to the requirement of consideration of finite parameters in the analysis, finding out the exact eigenvalue distribution of $\hat{\mathbf{R}}_{\mathbf{Y}}(N)$ becomes complicated. This makes the choice of the threshold difficult for SS purpose and the performance of SS algorithms becomes sensitive to the choice of threshold at low values of SNR. Although various Tracy-Widom (TW) distribution based approaches have been proposed in [8] and [24] for accounting the random nature of SCN of finite matrices, we are interested in analyzing noise correlation effect on MP based asymptotic methods in this paper. 
Under $H_{0}$ hypothesis, the received signal matrix $\mathbf{Y}$ from (4) and (6) can be written as:

$$
\mathbf{Y}=\hat{\mathbf{Z}}=\boldsymbol{\Theta}^{1 / 2} \mathbf{Z}
$$

In this noise only case, $\hat{\mathbf{R}}_{\mathbf{Y}}(N)$ becomes equal to $\hat{\mathbf{R}}_{\hat{\mathbf{Z}}}(N)$ and can be written as:

$$
\hat{\mathbf{R}}_{\mathbf{Y}}(N)=\hat{\mathbf{R}}_{\hat{\mathbf{Z}}}(N)=\boldsymbol{\Theta}^{1 / 2} \mathbf{Z} \mathbf{Z}^{H} \boldsymbol{\Theta}^{1 / 2}
$$

$\hat{\mathbf{R}}_{\mathbf{Y}}(N)$ converges to $\mathbf{R}_{\mathbf{Y}}$ for $N \rightarrow \infty$ [24] and asymptotic analysis still holds true for large values of $N$ [13]. Furthermore, $\hat{\mathbf{R}}_{\mathbf{Z}}(N)=\frac{1}{N} \mathbf{Z} \mathbf{Z}^{H}$ is nearly a Wishart random matrix [11] under white noise scenarios but is no longer a Wishart random matrix in correlated noise scenarios. To calculate the threshold for SS purpose, we need the support of a.e.p.d.f. of $\mathrm{Y}$, namely, $\lambda_{\max }$ and $\lambda_{\min }$. Due to noncommutative nature of random matrices, it is not straightforward to calculate the eigenvalue distribution of $\mathbf{Y}$ by knowing the eigenvalue distribution of $\Theta$ and $\mathbf{Z}$. Using free probability analysis, the asymptotic eigenvalue distribution of $\frac{1}{N} \mathbf{Y} \mathbf{Y}^{H}$ can be calculated by applying the $\Sigma$ transform [11].

$$
\Sigma_{\mathbf{Y}}(z)=\Sigma_{\boldsymbol{\Theta}}(z) \Sigma_{\mathbf{Z}}(z)
$$

where $\Sigma_{\Theta}$ and $\Sigma_{\mathbf{Z}}$ are the $\Sigma$ transforms of the densities of eigenvalues of $\boldsymbol{\Theta}$ and $\frac{1}{N} \mathbf{Z} \mathbf{Z}^{H}$ respectively. Since $\boldsymbol{\Theta}$ is a square matrix, $\boldsymbol{\Theta}^{1 / 2} \mathbf{Z} \mathbf{Z}^{H} \boldsymbol{\Theta}^{1 / 2}$ and $\boldsymbol{\Theta} \mathbf{Z} \mathbf{Z}^{H}$ have identical eigenvalues [11]. Since the R transform of density of eigenvalues of $\mathbf{Z} \mathbf{Z}^{H}$ is $\Sigma_{\mathbf{Z}}(z)=\frac{1}{z+\beta}$ [11], $\Sigma_{\mathbf{Y}}(z)$ can be written as:

$$
\Sigma_{\mathbf{Y}}(z)=\Sigma_{\boldsymbol{\Theta}}(z) \frac{1}{z+\beta}
$$

The $\Sigma$ transform of corresponding asymptotic eigenvalue distribution $\Sigma_{\Theta}(z)$ can be obtained by considering a proper model for noise correlation. The asymptotic density of eigenvalues of $\Theta$ can be described as a tilted semicircular law [22], which is a close approximation for the exponential model and is analytically tractable. This density can be described using the following theorem.

Theorem 3.2: [22] Let $\Theta$ be a positive definite matrix which is normalized as: $(1 / M) \operatorname{trace}\{\boldsymbol{\Theta}\}=1$, and whose asymptotic spectrum has the p.d.f.

$$
f_{\Theta}(\lambda)=\frac{1}{2 \pi \mu \lambda^{2}} \sqrt{\left(\frac{\lambda}{\sigma_{1}}-1\right)\left(1-\frac{\lambda}{\sigma_{2}}\right)}
$$

with $\sigma_{1} \leq \lambda \leq \sigma_{2}$ and $\mu=\frac{\left(\sqrt{\sigma_{2}}-\sqrt{\sigma_{1}}\right)^{2}}{4 \sigma_{1} \sigma_{2}}$. If $\mathbf{F}$ is an $M \times N$ standard Gaussian matrix as defined in theorem 1, then as $M, N \rightarrow \infty$ with $\frac{N}{M} \rightarrow \beta$, the asymptotic distribution of $\mathbf{W}=\boldsymbol{\Theta}^{1 / 2} \mathbf{F} \mathbf{F}^{H} \boldsymbol{\Theta}^{1 / 2}$ has the following p.d.f.

$$
f_{\mathbf{W}}(\lambda)=(1-\beta)^{+} \delta(\lambda)+\frac{\sqrt{(\lambda-\tilde{a})^{+}(\tilde{b}-\lambda)^{+}}}{2 \pi \lambda(1+\lambda \mu)}
$$

where

$$
\begin{aligned}
& \tilde{a}=1+\beta+2 \mu \beta-2 \sqrt{\beta} \sqrt{(1+\mu)(1+\mu \beta)} \\
& \tilde{b}=1+\beta+2 \mu \beta+2 \sqrt{\beta} \sqrt{(1+\mu)(1+\mu \beta)}
\end{aligned}
$$

The parameters $\tilde{a}$ and $\tilde{b}$ correspond to $\lambda_{\min }$ and $\lambda_{\max }$ respectively and the ratio $\tilde{b} / \tilde{a}$ defines the SCN of $\mathbf{W}$.

The degree of noise correlation is related to the eigenvalue spread of $\Theta$ i.e., a zero eigenvalue spread corresponds to a zero-correlation model $\Theta=\mathbf{I}_{M}$ and higher spreads are associated with higher correlation modes. In (15), $\mu$ is a parameter that controls the degree of noise correlation and varies the support of the distribution i.e., for $\mu=0, \tilde{a}=a$ and $\tilde{b}=b$. Low values of $\mu$ correspond to the low degree of correlation and high values of $\mu$ correspond to the high degree of noise correlation. The p.d.f $f_{\Theta}(\lambda)$ is bounded with the support $\left[\sigma_{1}, \sigma_{2}\right]$. The density of eigenvalues of $\mathbf{W}$ with its components given by (15) has the support $[1-\rho / 1+\rho, 1+\rho / 1-\rho]$. Thus the bounds of exponential correlation function can be fixed by setting $\frac{\sigma_{2}}{\sigma_{1}}=\left(\frac{1+\rho}{1-\rho}\right)^{2}$ [22] so that the distribution in (15) follows the same eigenvalue spread as the exponential model. This is equivalent to fixing the value of $\mu$ in terms of $\rho$ as:

$$
\mu=\frac{\rho^{2}}{1-\rho^{2}}
$$

Furthermore, the $\mathrm{SCN}$ is related to $\rho$ with the following relation:

$$
\mathrm{SCN}=\frac{1+\rho}{1-\rho}
$$

The value of $\mu$ in a practical cognitive receiver can be determined based on the value of $\rho$, which can be determined from FS rate based on some empirical model constructed from measurements. In this paper, we employ a simple linear model to study the relation of noise correlation with the FS rate (see Section IV). It can be noted that MP law can be used as a theoretical prediction under $H_{0}$ hypothesis with white noise [13]. The support of the eigenvalues of the sample covariance matrix under the $H_{0}$ hypothesis is finite independently of the distribution of the noise. To decide the absence or presence of signal under white noise, the deviations of distribution of eigenvalues from the normal bounds $a$ and $b$ of MP law can be used. If the eigenvalues appear outside these bounds, then it can be decided that there is presence of PU signal and if all the eigenvalues lie within the bounds of MP law, it can be decided that there is absence of PU signal. In case of noise correlation, the bounds of eigenvalue distribution of sample covariance matrix become different than the bounds obtained in white noise case and MP law no longer applies. The new bounds $(\tilde{a}, \tilde{b})$ depend on the noise correlation parameter $\mu$. We present the sensing with new bounds for FS scenario in the following section.

\section{Proposed Decision Statistic For FS Scenario}

Sampling rate at the $\mathrm{CR}$ receiver can be varied from the symbol rate to some order of the symbol rate and the effect of sampling rate on the sensing performance can be evaluated by varying the correlation effect. The parameter $\mu$ defined in the above section depends on the sampling rate applied at the $\mathrm{CR}$ receiver since noise correlation increases along with 
the sampling rate. Let us consider that both noise distribution and noise variance are unknown to the detector to reflect the practical scenario. It can be noted that the value of SCN under $H_{0}$ hypothesis does not depend on the noise variance since it is the ratio of the maximum and the minimum eigenvalue. Under white noise scenario, the decision is made on the basis of following equation [13]:

$$
\text { decision }= \begin{cases}H_{0}, & \text { if } \mathrm{SCN} \leq \frac{b}{a} \\ H_{1}, & \text { otherwise }\end{cases}
$$

Similar decision process can be applied for correlated noise scenario and we propose the following decision rule for making decision about the presence of the PU in the presence of noise correlation.

$$
\text { decision }= \begin{cases}H_{0}, & \text { if } \mathrm{SCN} \leq \frac{\tilde{b}}{\tilde{a}} \\ H_{1}, & \text { otherwise }\end{cases}
$$

When FS rate $M$ is applied at the $\mathrm{CR}, M$ rows of sample covariance matrix become correlated. The value $M=1$ corresponds to symbol rate sampling and correlation coefficient $\rho=0$. The relation between the parameters $\rho$ and $M$ is considered as a simple linear model ${ }^{1}$ as shown below

$$
\rho=\varepsilon\left(\frac{1}{\beta}-\frac{1}{N}\right)
$$

where $\varepsilon$ is a parameter defining the slope of the linear dependence.

\section{NumericAl RESUlts}

In this section, firstly, the performance of eigenvalue based sensing is studied in the presence of noise correlation with proposed decision bounds and then FS based SS is studied with numerical evaluation. The ratio of correct sensing is used as a performance metric to analyze the performance and it is defined as the ratio of number of correct sensing to the number of total considered iterations under both hypotheses. In other words, this metric defines the probability of correct decisions under both hypotheses. The number of iterations considered in the presented simulation results were $10^{3}$. The performance of the proposed sensing scheme has been analyzed in time varying Rayleigh fading channel. We consider the white and correlated noise scenarios in the numerical analysis. In case of white noise scenarios, it has been noted that the eigenvalue distribution of received signal's covariance matrix follows the MP law and the distribution is limited to the bounds given by the MP law as shown in [13]. Therefore, the decision rule in (19) is used for sensing the presence of the PU. However, the eigenvalue distribution breaks into two parts in the presence of noise correlation (Fig. 2, [21]) and the decision rule in (20) with the proposed sensing threshold is considered. The ratio of correct sensing versus SNR for $\rho=0.5, \beta=1 / 6$ is depicted in Fig. 1. From the figure, it can be observed that sensing with (20) outperforms than sensing with (19) in the presence of noise correlation.

${ }^{1}$ More exact relation models can be acquired through measurements on the CR equipment.

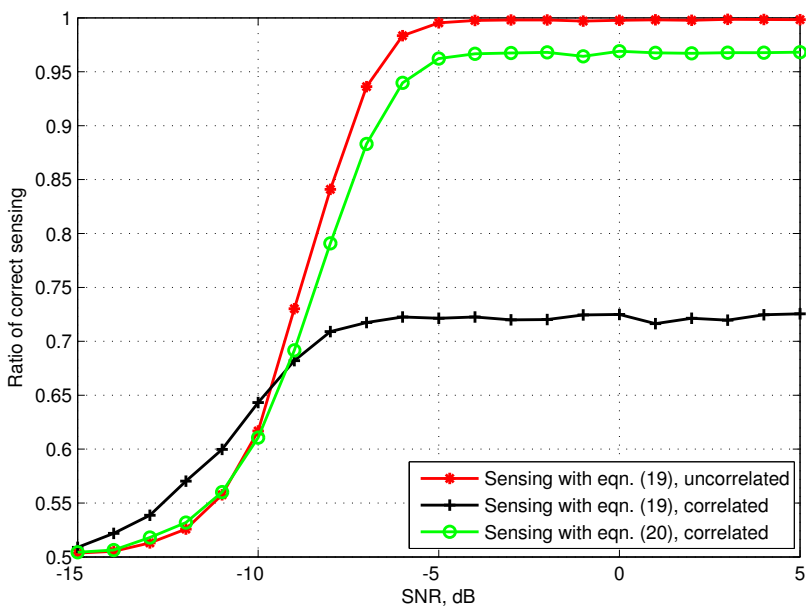

Fig. 1: Sensing performance versus SNR with (19) and (20) $(\beta=1 / 6, \rho=0.5, N=60)$

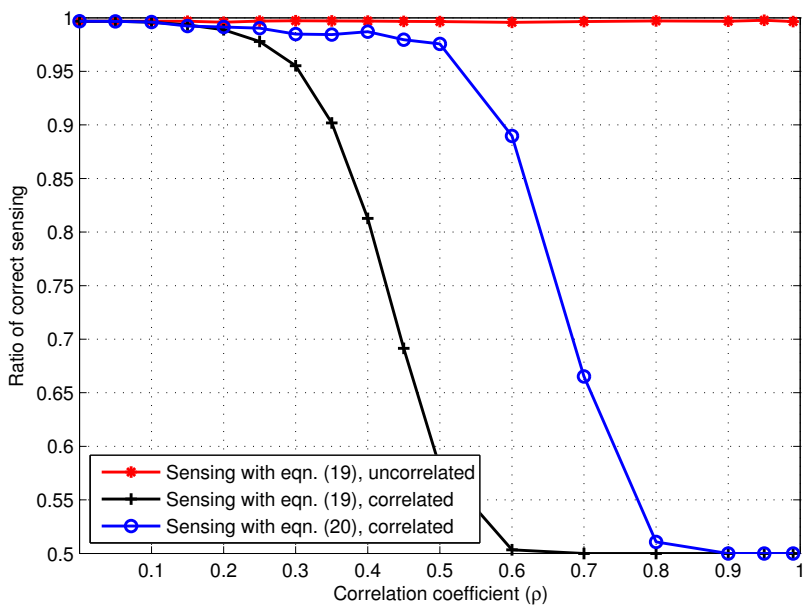

Fig. 2: Sensing performance versus correlation coefficient in presence of white noise and correlated noise $(\mathrm{SNR}=-6 \mathrm{~dB}, \beta=1 / 6, N=60)$

Figure 2 shows the sensing performance versus correlation coefficient at SNR value of $-6 \mathrm{~dB}$ and $\beta=1 / 6$ and it can be noted that with increased amount of noise correlation, the sensing with (19) decreases drastically and sensing with (20) provides better performance up to a certain value of correlation. Moreover, it has been noted that new bounds also do not provide better sensing at high correlation region. This is due to the fact that the threshold increases and the asymptotic eigenvalue support of $H_{1}$ is subsumed in the one of $H_{0}$ at this region.

Figure 3 shows the ratio of correct sensing versus FS rate $(M)$ for $\varepsilon=3.5$. The FS rate has been increased from 1 to 11 and noise correlation coefficient has been calculated using (21) for different values of $M$. It can be noted that the sensing performance increases with FS rate for white noise scenario. However, at the same time, noise becomes correlated due to FS and increasing the sampling rate does not monotonically increase the performance. From Fig. 3, it can be noted that for $N=60, \mathrm{SNR}=-5 \mathrm{~dB}$, the performance increases up to $\mathrm{FS}$ rate $M=8$ and for $M>8$, the sensing with (20) saturates. It can be observed that increasing sampling rate enhances the 


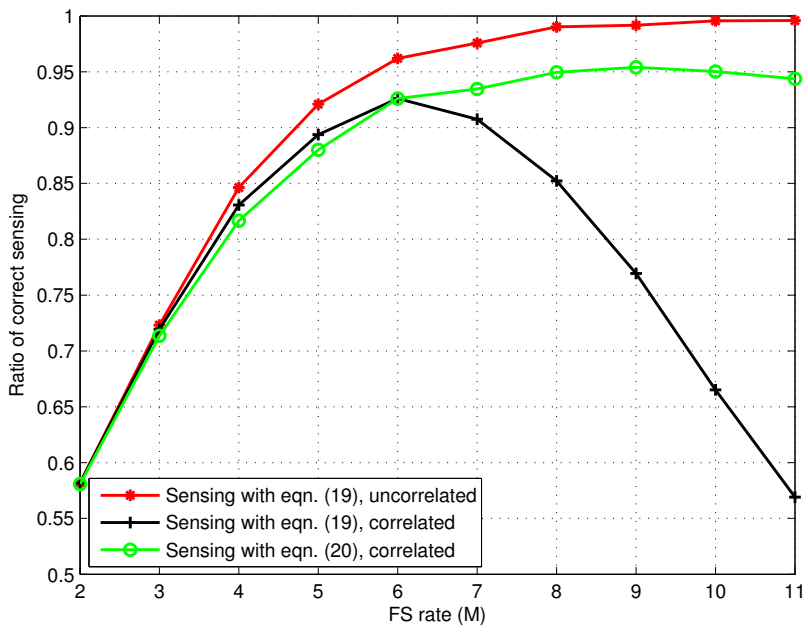

Fig. 3: Sensing performance versus FS rate $(N=60, \mathrm{SNR}=-5 \mathrm{~dB})$

sensing performance up to a certain FS rate, however, this also increases the complexity in the receiver. Thus it can be concluded that optimum sampling rate should be chosen at the receiver without increasing further complexity since larger rate does not enhance the performance due to the effect of noise correlation.

\section{Conclusion}

In this paper, a fractional sampling based SS technique has been studied in the presence of noise correlation using asymptotic RMT. The detailed analysis is carried out under noise only case and new bounds are proposed for improved sensing in correlated noise scenarios. From the simulation results, it has been observed that the proposed new threshold can be used for improved sensing performance in correlated noise scenarios. Furthermore, it has been noted that SS efficiency increases with FS rate up to a certain limit and it does not provide advantage beyond this limit. Therefore, it can be concluded that an optimal operating point for the FS rate should be selected to maintain a good trade-off between performance and complexity. The analysis of the effect of correlation in multiple antenna systems and multiple user environments as well as the analysis of correlation effect using RMT approaches other than the MP based methods will be future work in this area.

\section{ACKNOWLEDGEMENT}

This work was supported by the National Research Fund, Luxembourg under AFR (Aids Training-Research) grant for $\mathrm{PhD}$ project (Reference 3069102) on "Spectrum Sensing, Resource Allocation and Resource Management Strategies for Satellite Cognitive Communications", under the CORE project "CO2SAT: Cooperative and Cognitive Architectures for Satellite Networks" and COST action IC0902.

\section{REFERENCES}

[1] K. Patil, R. Prasad, and K. Skouby, "A survey of worldwide spectrum occupancy measurement campaigns for cognitive radio," in Int. Conf. Devices and Commun., Feb. 2011, pp. $1-5$.
[2] A. Goldsmith, S. Jafar, I. Maric, and S. Srinivasa, "Breaking spectrum gridlock with cognitive radios: An information theoretic perspective," Proc. of the IEEE, vol. 97, no. 5, pp. 894 -914, May 2009.

[3] S. K. Sharma, S. Chatzinotas, and B. Ottersten, "Satellite cognitive communications: Interference modeling and techniques selection," in Proc. 6th ASMS/SPSC Conf., Sept. 2012, pp. 111-118.

[4] T. Yucek and H. Arslan, "A survey of spectrum sensing algorithms for cognitive radio applications," IEEE Communications Surveys Tutorials, vol. 11, no. 1, pp. $116-130$, quarter 2009.

[5] S. K. Sharma, S. Chatzinotas, and B. Ottersten, "Exploiting polarization for spectrum sensing in cognitive SatComs," in Proc. 7th Int. Conf. CROWNCOM, June 2012, pp. 36-41.

[6] S. K. Sharma, S. Chatzinotas, and B. Ottersten, "Spectrum sensing in dual polarized fading channels for cognitive SatComs," in Proc. IEEE GLOBECOM Conf., Dec. 2012, pp. 3443-3448.

[7] R. Tandra and A. Sahai, "SNR walls for signal detection," IEEE J. Sel. Topics Signal Process, vol. 2, no. 1, pp. 4 -17, Feb. 2008.

[8] Y. Zeng and Y. chang Liang, "Eigenvalue-based spectrum sensing algorithms for cognitive radio," IEEE Trans. Commun., vol. 57, no. 6, pp. $1784-1793$, June 2009

[9] W. Zhang, G. Abreu, M. Inamori, and Y. Sanada, "Spectrum sensing algorithms via finite random matrices," IEEE Trans. Commun., vol. 60, no. 1, pp. $164-175$, Jan. 2012.

[10] A. Kortun, T. Ratnarajah, M. Sellathurai, C. Zhong, and C. Papadias, "On the performance of eigenvalue-based cooperative spectrum sensing for cognitive radio," IEEE J. Sel. Topics Signal Process, vol. 5, no. 1, pp. $49-55$, Feb. 2011.

[11] A. M. Tulino and S. Verdu, "Random matrix theory and wireless communications," Foundations and Trends in Communications and Information Theory, vol. 1, no. 1, pp. 1-182, 2004.

[12] R. Couillet and M. Debbah, Random Matrix Methods for Wireless Communications, 1st ed. Cambridge University Press, 2011.

[13] L. Cardoso, M. Debbah, P. Bianchi, and J. Najim, "Cooperative spectrum sensing using random matrix theory," in 3rd Int. Symp. Wireless Pervasive Computing, May 2008, pp. $334-338$.

[14] M. Matthaiou, M. Mckay, P. Smith, and J. Nossek, "On the condition number distribution of complex wishart matrices," IEEE Trans. Commun., vol. 58, no. 6, pp. $1705-1717$, June 2010.

[15] C. Tepedelenlioglu and R. Challagulla, "Low-complexity multipath diversity through fractional sampling in OFDM," IEEE Trans. Signal Process., vol. 52, no. 11, pp. 3104 - 3116, Nov. 2004.

[16] Y. Imaoka and Y. Sanada, "Experimental investigation of sampling rate selection with fractional sampling for IEEE802.11b WLAN system," in IEEE 70th Veh. Technol. Conf., Sept. 2009, pp. 1 -5.

[17] B. Li, Q. Wang, G. Lu, Y. Chang, and D. Yang, "Linear MMSE frequency domain equalization with colored noise," in IEEE 66th Veh. Technol. Conf., Sept. 30-oct. 3 2007, pp. $1152-1156$.

[18] A. Vasilopoulos, G. Vitzilaios, G. Theodoratos, and Y. Papananos, "A low-power wideband reconfigurable integrated active RC filter with 73 dB SFDR," IEEE J. Solid-State Circuits, vol. 41, no. 9, pp. 1997 -2008, Sept. 2006.

[19] H. Shin and Y. Kim, "A CMOS active RC low-pass filter with simultaneously tunable high and low cutoff frequencies for IEEE 802.22 applications," IEEE Trans. on Circuits and Systems II: Express Briefs, vol. 57, no. 2, pp. $85-89$, Feb. 2010.

[20] S. Loyka, "Channel capacity of MIMO architecture using the exponential correlation matrix," IEEE Commun. Letters, vol. 5, no. 9, pp. 369 -371, Sept. 2001.

[21] S. Chatzinotas, M. Imran, and R. Hoshyar, "On the multicell processing capacity of the cellular MIMO uplink channel in correlated Rayleigh fading environment," IEEE Trans. Wireless Commun., vol. 8, no. 7, pp. $3704-3715$, July 2009.

[22] X. Mestre, J. Fonollosa, and A. Pages-Zamora, "Capacity of MIMO channels: asymptotic evaluation under correlated fading," IEEE J. Sel. Areas Commun., vol. 21, no. 5, pp. 829 - 838, June 2003.

[23] S. K. Sharma, S. Chatzinotas, and B. Ottersten, "Eigenvalue based sensing and SNR estimation for cognitive radio in presence of noise correlation," Submitted to IEEE trans. in Veh. Techn., 2012. [Online]. Available: http://arxiv.org/abs/1210.5755

[24] F. Penna, R. Garello, and M. Spirito, "Cooperative spectrum sensing based on the limiting eigenvalue ratio distribution in Wishart matrices," IEEE Commun. Letters, vol. 13, no. 7, pp. 507 -509, July 2009. 